\title{
Apropiación social del conocimiento y bien común: una propuesta para evaluar el impacto de la educación superior
}

\section{Social appropriation of knowledge and common good: a proposal to evaluate the impact of higher education}

\author{
Juan José Torrente Rocha ${ }^{1}$ \\ Vicente Manzano Arrondo? \\ María del Carmen Rodríguez Morilla ${ }^{3}$
}

(2)

Recepción: 31/08/2020
(-)

Aprobación: 25/09/2020
(.)

Publicación: 18/12/2020

\section{Para citar este artículo:}

Torrente Rocha, J. J., Manzano Arrondo, V., \& Rodríguez Morilla, M. C. (2020). Apropiación social del conocimiento y bien común: una propuesta para evaluar el impacto de la educación superior. Indagare, (8), 46-57. https://doi.org/10.35707/indagare/805

\section{(c) $(9)$}

\footnotetext{
${ }^{1}$ Grupo de investigación GESS, Universidad de Ibagué, Colombia. ORCID: 0000-0002-4142-1560. Correo electrónico: juan. torrente@unibague.edu.co

${ }^{2}$ Departamento de Psicología Experimental, Universidad de Sevilla, España. ORCID: 0000-0003-0456-8480. Correo electrónico: vmanzano@us.es

${ }^{3}$ Programa de Economía Aplicada, Universidad de Sevilla, España. ORCID: 0000-0002-5706-5935. Correo electrónico: cmorilla@us.es
} 


\section{Resumen}

La evaluación es un proceso determinante de la calidad en las instituciones de educación superior, sin embargo, identificar cuál es el impacto que la universidad tiene sobre la comunidad extendida es todavía un reto más difícil de superar. El objetivo de esta investigación es diseñar una evaluación de impacto de un programa de educación superior sobre la región, basada en indicadores de apropiación social del conocimiento y bien común. Se utiliza una metodología cualitativa a partir de la creación y adaptación de indicadores descriptivos que brindan un marco contextual e interpretativo de los resultados. Al final se obtiene una propuesta confiable y novedosa, que no pretende una aplicación homogeneizante de indicadores, sino un ejercicio emancipador de reconocimiento para la mejora continua.

\section{Abstract}

Evaluation is a determining process of quality in higher education institutions. However, identifying the impact that the university has on the extended community is still a more difficult challenge to overcome. The aim of this research is to design an impact evaluation of a higher education program in the region, based on indicators of social appropriation of knowledge and common good. A qualitative methodology is used from the creation and adaptation of descriptive indicators that provide a contextual and interpretative framework of the results. At the end, a reliable and groundbreaking proposal is obtained, which does not intend a homogenizing application of indicators, but rather an emancipatory exercise of recognition for continuous improvement.

\footnotetext{
Palabras claves: Educación superior, evaluación de impacto, apropiación social del conocimiento, bien común, universidad. Key words: Higher education, impact evaluation, social appropriation of knowledge, common good, university.
}

\section{Introducción}

La medición del impacto de los programas académicos, en esencia, no cuenta con procesos para tal fin, más allá de la cuantificación de egresados, cursos y capacitaciones realizadas, proyectos de investigación en curso y finalizados, sitios de prácticas en convenio activo y otras acciones puntuales que permiten establecer una cifra de resultado y cobertura. De acuerdo con el Informe de Renovación de la Acreditación, el Programa de Psicología de 
la Universidad de Ibagué da por hecho la "generación de impacto a través de la formación de psicólogos que responden a las necesidades de las organizaciones públicas y privadas" (Universidad de Ibagué, 2015, p. 56), afirmación que pierde validez al identificar como necesidad de mejoramiento más adelante, en el mismo documento, 1) la evaluación del impacto de los egresados sobre el medio social y académico, 2) la identificación del impacto generado por el material docente y 3) la medición del impacto de la investigación y la extensión en el medio; tres aspectos que resume el documento como la acción de mejora: "realizar estudios a partir de proyectos de investigación o trabajos de grado para identificar las necesidades y requerimientos del entorno laboral y a evaluar el impacto del programa" (p. 95).

En resumen, se asume que se está cumpliendo con la misión institucional desconociendo con evidencias y sustentos objetivos el impacto generado por los procesos académicos sobre el desarrollo de la región. En este sentido surge la pregunta de investigación de ¿cómo diseñar una evaluación de impacto de un programa de educación superior sobre el desarrollo de la región? Y más allá de eso, ¿cómo aportar a la gestión universitaria a través de una herramienta de evaluación flexible a las condiciones propias de cada programa académico? Estas y otras preguntas surgen a la luz de la problemática identificada no solo en el contexto de la universidad regional, sino en el de todas las universidades a falta de instrumentos, indicadores, herramientas o mecanismos para evaluar impacto en la educación superior de una manera sencilla y deshomogeneizante.

De acuerdo con lo anterior, tres elementos hacen parte de esta propuesta evaluativa: 1) la identificación del tipo de modelo universitario que contextualiza la interpretación de los resultados; 2) la evaluación del nivel de apropiación social del conocimiento en estudiantes y graduados; 3 ) la medición de aportes al bien común desde la perspectiva de todos los grupos de interés relacionados con el programa (estudiantes, graduados, docentes, administrativos y empresarios). En los siguientes párrafos se exponen las características de diseño, los resultados y el análisis del impacto del Programa de Psicología de la Universidad de Ibagué sobre el desarrollo de la región, como caso de estudio.

La evaluación de impacto se entiende como el proceso orientado a medir los resultados de las intervenciones en calidad, cantidad y extensión según las reglas preestablecidas (Scoppetta, 2006). La función principal es permitir comparar el grado de realización alcanzado con el grado de realización esperado o, en otras palabras, comparar la planeación con el resultado de la ejecución (Mestre, 2016). Con base en Robin (2016) consiste en comprobar la realidad de la gestión después de un determinado plazo de tiempo, que corresponde al tiempo estimado para que el cambio o los cambios puedan 
tener lugar, es decir que la evaluación del impacto es posible después de medir el alcance de resultados inmediatos y de los efectos provocados en el mediano plazo. El impacto se mide entonces luego de sistematizar los resultados y efectos de la gestión.

Como se trata de un ejercicio de medición, el objeto por medir debe tener unas variables cuantitativas y cualitativas que sean factibles de traducir en indicadores (Mestre, 2016). Estas variables pueden ser cuantitativas y cualitativas en términos generales, y de manera específica, pueden ser contextuales (modulan la interpretación de la evaluación teniendo en cuenta la situación en que se insertan) de entrada (describen los medios que están a disposición como recursos físicos, materiales, de personal, etc.), de proceso (relativas al desarrollo del programa evaluado, metodología) y de producto (ponen de manifiesto el logro de los objetivos a nivel de capacitación, inserción laboral, etc.).

Siguiendo a Fernández (2013), el problema de los indicadores se centra en el tipo de unidades de información sobre las cuales se justifica la necesidad de emitir un juicio de valor para tomar decisiones de mejora. De esta manera, Biencito y Carballo, citados por Fernández (2013), definen cuatro tipos de dimensiones que explican el impacto de la formación y que pueden servir de indicadores: 1) satisfacción: índice de calidad y correcto funcionamiento del programa; 2) valor añadido: incremento de conocimientos tras participar en la formación y su posterior transferencia al puesto de trabajo; 3) mejora del estatus profesional: incremento de las competencias profesionales; 4) mejora organizacional: incremento de las competencias institucionales. La medición del impacto en esta oportunidad se centra en las tres primeras dimensiones y deja la cuarta para la evaluación de desempeño profesoral, como referencia del mejoramiento en las competencias institucionales.

Dado que la propuesta se basa en el diseño de unos indicadores de apropiación social de conocimiento y del bien común, es pertinente mencionar algunos lineamientos teóricos de estos conceptos. Según lo dispuesto por Colciencias (2010) la apropiación social del conocimiento es el:

Proceso intencionado de comprensión e intervención en las relaciones entre ciencia, tecnología y sociedad, para ampliar las dinámicas de generación, circulación y uso del conocimiento científico-tecnológico entre los sectores académicos, productivos, estatales, incluyendo activamente a las comunidades y grupos de interés de la sociedad civil. (s.p.).

Sus cuatro componentes de participación ciudadana, comunicación, intercambio y gestión del conocimiento conllevan pensar que el impacto desde la apropiación del saber implica adentrarse en los terrenos de una educación transformadora más que de 
cobertura, que empodera en cada una de sus funciones a los participantes en su rol de gestores sobre el desarrollo, el tejido social y la sostenibilidad. La determinación de ítems en esta propuesta se basa en el aporte de indicadores cualitativos de Daza-Caicedo et al. (2017) en la medición de la apropiación social de la ciencia y la tecnología (ASCyT) para el caso puntual de Maloka (Museo Interactivo en Bogotá, Colombia) junto con el Observatorio de Ciencia y Tecnología.

En principio, depende de la agencia universitaria lograr que la comunidad en general pueda apropiarse del proceso de aprendizaje, no obstante, la forma como se desarrollen sus estrategias depende en gran medida de cómo los docentes, estudiantes, administrativos y comunidad en general, desde su rol de receptores-emisores, perciben el modelo de gestión institucional, puesto que las percepciones como principal componente de las actitudes permitirán o no la acción.

Tras revisar el planteamiento de Manzano-Arrondo \& Torrego (2009) es factible diferenciar tres modelos de universidad. El modelo A se basa en el concepto de la universidad como una entidad autónoma que se ocupa de la concepción y transmisión del conocimiento con el fin de aportar a la formación integral de personas efectivas e intelectualmente capaces. El modelo B enfatiza en la reproducción de conocimiento y la formación como proceso por el cual los graduados adquieren competencias y habilidades concretas para desempeñar su quehacer laboral. El modelo $\mathrm{C}$ toma de los anteriores modelos la necesidad que existe para la formación integral del conocimiento, aunque pone principal atención en orientar la gestión educativa hacia la solución de los grandes problemas de la sociedad y de los nuevos problemas del planeta. No solo desde la generación de conocimiento pertinente, sino también desde la intervención basada en el conocimiento generado junto con los principales actores de una ciudadanía comprometida.

El modelo C da paso al modelo de Unidades de Acción Comprometida (UAC), expuesto por Manzano-Arrondo (2011) y Manzano-Arrondo \& Suárez (2015), que puede denominarse como un cuarto modelo caracterizado por una intención emancipadora que emerge de la necesidad de balancear el protagonismo de las tres funciones de la universidad hacia el bien común. Basadas en la investigación acción participativa y en el aprendizaje de servicio, las UAC potencian la ciencia y la academia para organizar unidades de aprendizaje y transformación social en sinergia con el tercer sector, hecho que a la vez aumenta la utilidad pública de las instituciones de educación superior y permite una formación mejor instalada en la complejidad de los contextos reales.

El tercer elemento constitutivo de la propuesta de evaluación es la implementación de una herramienta de auditoría empresarial ajustada a la naturaleza de la universidad: el balance del bien común (BBC). Los indicadores de bien común se basan en la perspectiva 
INDAGA3E e-ISSN: 2357-5042 • Número 8 (2020) • Universidad de Ibagué • doi: https://doi.org/10.35707/indagare/805

de Christian Felber para quien las organizaciones de los distintos sectores deben tener métricas balanceadas entre las ganancias económicas o de eficiencia y los aportes al bien común, entendidos como "el incentivo de cualidades y valores del comportamiento que contribuyen al éxito de las relaciones humanas y ecológicas: dignidad humana, solidaridad y justicia social, sostenibilidad ambiental y transparencia" (Felber, 2012, p. 62).

Las contribuciones a esos valores se observan por medio de la herramienta balance del bien común, que consiste en una matriz cruzada de principios de acción con los grupos de interés que componen, en este caso, el programa de psicología: representantes de empresarios como proveedores, personal administrativo de la universidad en calidad de inversores, profesores en el rol de los empleados, estudiantes como clientes y graduados como representantes del ámbito social.

\section{Materiales y métodos}

El diseño de una evaluación de impacto con las características anteriores corresponde a una investigación cualitativa con apoyo estadístico para el análisis de validez y fiabilidad de los instrumentos. El tipo de estudio es descriptivo con diseño transversal, que de acuerdo con Hernández, Fernández \& Baptista (2018) y Teddlie \& Tashakkori (2009), considera el fenómeno estudiado y sus componentes midiendo conceptos y definiendo variables para después establecer relaciones entre las mismas en un momento de medición particular. Las herramientas que componen la propuesta de evaluación de impacto equivalen a un estudio de tipo tecnológico dado que "utiliza conocimientos existentes, procedentes de la investigación aplicada o de la experiencia práctica para la generación de sistemas o servicios nuevos o mejoras" (Escorsa \& Valls, 2016, p. 69).

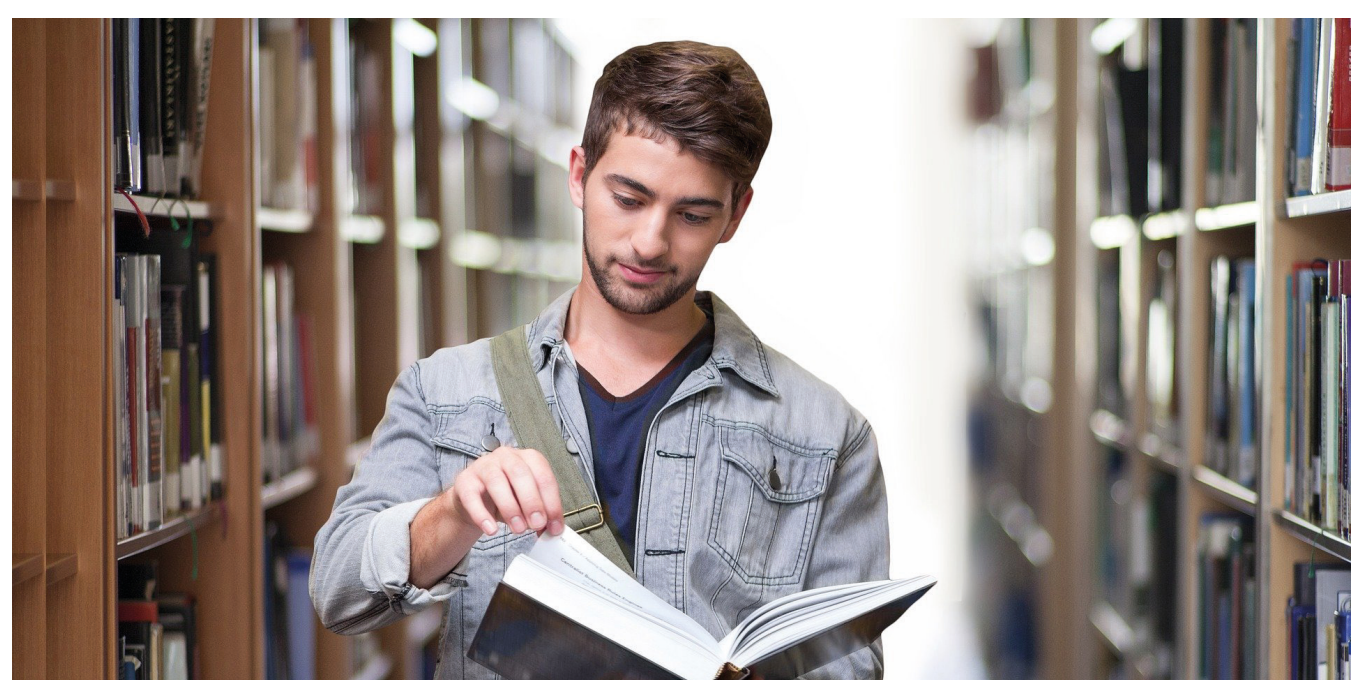


El procedimiento se puede describir de la siguiente manera: 1) selección de una muestra probabilística para las escalas y no probabilística para el balance de bien común; 2) aplicación de las escalas en salas de cómputo por medio de un enlace de distribución virtual a través de la plataforma QUALTRICS; 3) aplicación del balance de bien común a través de la conformación de grupos de discusión con cada grupo de interés; 4) recolección, sistematización y análisis de la información.

La aplicación de los instrumentos propuestos se realizó en el marco del proceso de autoevaluación con fines de renovación de la acreditación de alta calidad del Programa de Psicología de la Universidad de Ibagué. Para seleccionar los participantes se efectuó un muestreo estratificado que tuvo en cuenta a los estudiantes de segundo a décimo semestre para la medición de los indicadores de apropiación, impacto y la identificación del modelo de universidad (Tabla 1). La aplicación se llevó a cabo por medio de la plataforma QUALTRICS y los datos se analizaron a través del software estadístico SPSS versión 25. Fue utilizado un muestreo por conveniencia para la aplicación del balance del bien común en una dinámica participativa cuyos resultados se cuantificaron siguiendo una lógica propia.

Tabla 1. Identificación de la muestra

\begin{tabular}{|c|c|c|c|}
\hline & & Frecuencia & Porcentaje \\
\hline \multirow[t]{5}{*}{ Válidos } & Estudiante & 171 & 84,7 \\
\hline & Graduado & 16 & 7,9 \\
\hline & Docente & 9 & 4,5 \\
\hline & Catedrático & 6 & 3,0 \\
\hline & Total & 202 & 100,0 \\
\hline
\end{tabular}

Fuente: elaboración propia

Los instrumentos para la medición del impacto y la ASC solo se aplicaron al grupo de estudiantes y de graduados, estos últimos fueron contactados por medio de un enlace virtual. La fiabilidad de las escalas de ASC e impacto es alta, hecho que implica que ante una población similar los instrumentos pueden tener un comportamiento parecido (Tabla 2). 
INDAGA:ZE e-ISSN: 2357-5042 • Número 8 (2020) • Universidad de Ibagué • doi: https://doi.org/10.35707/indagare/805

Tabla 2. Análisis de fiabilidad de las escalas

\begin{tabular}{|c|c|c|c|c|}
\hline Escala & $\begin{array}{c}\text { Alfa de } \\
\text { Cronbach }\end{array}$ & $\begin{array}{c}\mathrm{N} .^{\circ} \text { de } \\
\text { elementos }\end{array}$ & Válidos & $\%$ \\
\hline $\begin{array}{l}\text { CENA-Apropiación social del } \\
\text { conocimiento }\end{array}$ & ,742 & 9 & 187 & 92,6 \\
\hline CEDI-Impacto & ,909 & 12 & 187 & 92,6 \\
\hline
\end{tabular}

Fuente: elaboración propia

La escala cuestionario de identificación del modelo de universidad (CIMU) es de tipo ordinal, por lo cual el análisis de fiabilidad se realizó a través de un procedimiento test-retest del que se obtuvieron datos similares en el comportamiento de los 16 ítems. El cálculo de los resultados se realizó a través del análisis de medias y la jerarquización de las mismas de mayor a menor.

\section{Resultados}

Los resultados demuestran una mayor inclinación hacia el modelo $\mathrm{C}$ de universidad. En específico se puede construir una definición de la Universidad de Ibagué reuniendo los resultados obtenidos de la siguiente manera: la Universidad de Ibagué es un centro de investigación, docencia, diseño e intervención al servicio de la construcción de conocimiento, que busca a través del manejo eficiente de los recursos, la exigencia de la calidad y el control de los procesos, formar personas autónomas que sean verdaderos agentes de cambio social, en el marco de la responsabilidad, el respeto por la diversidad y el compromiso.

Los resultados obtenidos en el CIMU establecen entonces el marco de referencia interpretativo y contextualizador de la evaluación de impacto sobre el nivel de apropiación social del conocimiento y el bien común. La muestra de estudiantes, de acuerdo con sus respuestas en la escala cuestionario de evaluación del nivel de apropiación (CENA), muestra tener sentimientos de productividad y capacidad, pero dicha capacidad parece estar asociada con áreas disciplinares a un nivel muy básico en comparación con el perfil de profesional deseado por el programa académico e identificado en el perfil de universidad CIMU, lo cual se respalda con los bajos resultados en las dimensiones de ASC: participación en el diseño de cambios sociales y la capacidad para crear innovaciones. 
INDAGA3E e-ISSN: 2357-5042 • Número 8 (2020) • Universidad de Ibagué • doi: https://doi.org/10.35707/indagare/805

Tabla 3. Resultados del CENA

\begin{tabular}{|c|c|c|c|c|c|c|c|c|c|}
\hline \multirow{2}{*}{$\begin{array}{l}\text { Dimensión } \\
\text { Indicadores }\end{array}$} & \multicolumn{2}{|c|}{$\begin{array}{l}\text { Participación } \\
\text { ciudadana en CteI }\end{array}$} & \multicolumn{3}{|c|}{ Comunicación } & \multicolumn{2}{|c|}{$\begin{array}{l}\text { Intercambio de } \\
\text { conocimiento }\end{array}$} & \multicolumn{2}{|c|}{$\begin{array}{l}\text { Gestión del } \\
\text { conocimiento }\end{array}$} \\
\hline & 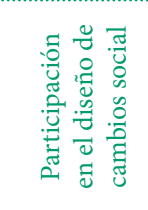 & 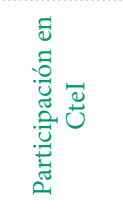 & 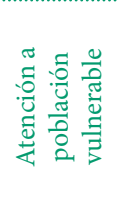 & 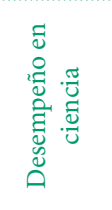 & 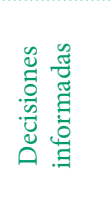 & 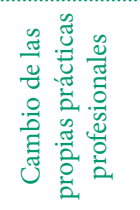 & 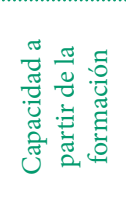 & 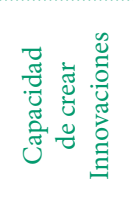 & 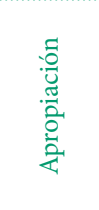 \\
\hline Válidos & 187 & 187 & 187 & 187 & 187 & 187 & 187 & 187 & 187 \\
\hline Perdidos & 15 & 15 & 15 & 15 & 15 & 15 & 15 & 15 & 15 \\
\hline Media & 2,59 & 2,68 & 2,61 & 2,59 & 2,35 & 2,67 & 2,55 & 2,66 & 2,55 \\
\hline Mediana & 2 & 3 & 3 & 3 & 2 & 3 & 3 & 3 & 3 \\
\hline Moda & 3 & 3 & 3 & 1 & 1 & 3 & 3 & 1 & 3 \\
\hline Desv. típ. & 1,203 & 1,192 & 1,25 & 1,443 & 1,254 & 1,105 & 1,141 & 1,387 & 1,132 \\
\hline
\end{tabular}

Fuente: elaboración propia

Los resultados dan cuenta de una población que en términos generales ha apropiado el conocimiento recibido a través de los estudios de un oficio profesional, no obstante, los niveles son moderados y fluctúan entre 2,35 y 2,68 (escala de 0 a 4). Tras la aplicación del instrumento cuestionario de evaluación del impacto (CEDI) que evalúa las dimensiones de generación de valor añadido, satisfacción con el programa académico y contribución a la mejora del estatus profesional, los resultados muestran promedios que van de 3,23, 3,12 y 3,09 respectivamente; esto se traduce en una percepción media-alta del impacto que genera el programa sobre el cumplimiento de las expectativas de ingreso, proceso y egreso. 
INDAGA?E e-ISSN: 2357-5042 • Número 8 (2020) • Universidad de Ibagué • doi: https://doi.org/10.35707/indagare/805

Tabla 4. Dimensiones e indicadores de impacto

\begin{tabular}{|c|c|c|c|c|c|c|c|c|c|c|c|c|}
\hline \multirow{2}{*}{$\begin{array}{l}\text { Dimensión } \\
\text { Indicadores }\end{array}$} & \multicolumn{3}{|c|}{$\begin{array}{c}\text { Participación } \\
\text { ciudadana en CteI }\end{array}$} & \multicolumn{3}{|c|}{ Comunicación } & \multicolumn{3}{|c|}{$\begin{array}{l}\text { Intercambio de } \\
\text { conocimiento }\end{array}$} & \multicolumn{3}{|c|}{$\begin{array}{l}\text { Gestión del } \\
\text { conocimiento }\end{array}$} \\
\hline & 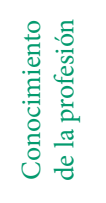 & 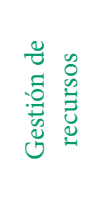 & 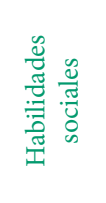 & 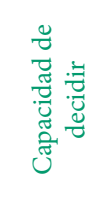 & 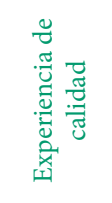 & 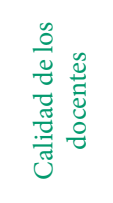 & 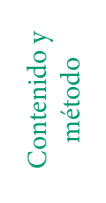 & 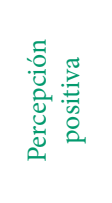 & $\begin{array}{l}\overrightarrow{\mathscr{U}} \\
\text {. }\end{array}$ & $\frac{\stackrel{8}{0}}{\text { : }}$ & 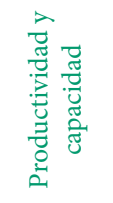 & 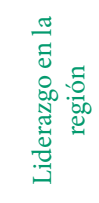 \\
\hline Válidos & 187 & 187 & 187 & 187 & 187 & 187 & 187 & 187 & 187 & 187 & 187 & 187 \\
\hline Perdidos & 15 & 15 & 15 & 15 & 15 & 15 & 15 & 15 & 15 & 15 & 15 & 15 \\
\hline Media & 3,28 & 3,11 & 3,26 & 3,3 & 3,04 & 3,12 & 2,94 & 3,39 & 2,94 & 2,94 & 3,29 & 3,19 \\
\hline Mediana & 3 & 3 & 3 & 3 & 3 & 3 & 3 & 3 & 3 & 3 & 3 & 3 \\
\hline Moda & 3 & 3 & 3 & 3 & 3 & 3 & 3 & 4 & 3 & 3 & 3 & 3 \\
\hline Desv. típ. & 0,678 & 0,663 & 0,679 & 0,653 & 0,638 & 0,648 & 0,716 & 0,658 & 0,697 & 0,697 & 0,588 & 0,633 \\
\hline
\end{tabular}

Fuente: elaboración propia

Las puntuaciones más bajas se encuentran relacionadas con la dificultad percibida para aumentar la red de contactos personales y profesionales, así como para aumentar la empleabilidad, entendida como la probabilidad de ser empleado en el mercado laboral. Por otra parte, se expone una percepción más positiva hacia la profesión, el aumento significativo en el conocimiento de la psicología y el mejoramiento de las habilidades sociales y el trabajo en equipo. Llama la atención la percepción de un aumento de la capacidad para tomar decisiones de forma sana y proactiva, en contravía con los resultados sobre la ASC frente a la capacidad de tomar decisiones informadas, situación que podría interpretarse como un aumento en la autonomía, pero no en el uso del conocimiento científico para decidir.

Finalmente, en la aplicación del balance participaron cinco muestras divididas de acuerdo con los grupos de interés que señalan la herramienta: grupo A. Proveedores: organizaciones, empresarios o grupos que ofrecen experiencias de práctica profesional a los estudiantes del programa (n: 5); grupo B. Directivos: funcionarios del programa que han ejercido cargos de dirección, auxiliares administrativos, coordinadores de centros de servicios (n: 7); grupo C. Docentes: grupo de profesores del programa académico en 
modalidad de planta y cátedra, y con participación en procesos de docencia, investigación y extensión social (n: 11); grupo D. Estudiantes: usuarios directos del servicio formativo del programa, son representantes estudiantiles quienes participan en el ejercicio (n: 9); y grupo E. Comunidad: personas en calidad de graduados del programa (n: 5).

La puntuación global del bien común expone unos aportes del $68.16 \%$, porcentaje que en una distribución normal indica un nivel alto de contribución, cifra que también refiere un amplio espectro de mejoramiento dirigido, en especial, a las dinámicas internas con los docentes y la sostenibilidad de las relaciones con el grupo de empresarios.

\section{Potencial uso}

En virtud del modelo de universidad, se puede decir que las funciones de formación, investigación y extensión aportan de manera moderada sobre la apropiación social del conocimiento y el bien común, sin embargo, es necesario hacer el análisis del impacto considerando una clara inconsistencia entre la percepción del perfil profesional y las mediciones efectuadas. Lo anterior tiene que ver con una percepción generalizada de un perfil deseado de psicólogo con énfasis en la gestión de cambios sociales, que se contradice con la percepción de capacidad para participar en el diseño de cambios sociales o en la creación de innovaciones basadas en ciencia y tecnología. Por esto no es suficiente evaluar el impacto de un programa de educación superior en términos de la formación de profesionales hábiles en su disciplina.

En últimas, una evaluación que mida el impacto a partir de apropiación social del conocimiento y los aportes al bien común, debe ser vista como una medición prospectiva de la calidad que, si bien sustenta las decisiones en términos de la planeación del mejoramiento, promueve, en especial, la participación conjunta entre la académica y la sociedad para legitimar su existencia de manera coherente y democrática.

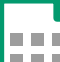

Ficha técnica del proyecto

Título del proyecto: Apropiación social del conocimiento y bien común: una propuesta para evaluar el impacto universitario sobre la región.

PRIT: Educación y bienestar para el desarrollo humano integral.

Código del proyecto: $19-495$-INT.

Palabras claves: Impacto, apropiación social, bien común.

Grupo de investigación: GESS.

Investigador principal: Juan José Torrente Rocha.

Correo electrónico: juan.torrente@unibague.edu.co 
INDAGAZEE e-ISSN: 2357-5042 • Número 8 (2020) • Universidad de Ibagué • doi: https://doi.org/10.35707/indagare/805

\section{Referencias}

Colciencias. (2010). Fortalecimiento en la producción de proyectos museológicos para la apropiación social de CTeI desarrollados por centros de ciencia. Anexo 3. Sobre la apropiación social de la ciencia, la tecnología y la innovación. Recuperado de https://n9.cl/e7e8

Daza-Caicedo, S., Maldonado, O., Moreno, P., Tafur-Sequera, M., Arboleda-Castrillón, T., Falla, S., \& Papagayo, D. (2017). Hacia la medición del impacto de las prácticas de apropiación social de la ciencia y la tecnología: propuesta de una batería de indicadores. História, Ciências, Saúde-Manguinhos, 24(1), 145-164. https://doi.org/10.1590/s0104-59702017000100004

Escorsa, P., \& Valls, J. (2016). Tecnología e innovación en la empresa. Barcelona, España: Ediciones UPC.

Felber, C. (2012). La economía del bien común. Barcelona, España: Ediciones Deusto.

Fernández, M. J. (2013). Evaluación de impacto para un cambio sostenible en las organizaciones educativas, Revista española de pedagogía, 71(254), 119-138. Recuperado de https://n9.cl/ljaig

Hernández, R., Fernández, C., \& Baptista, P. (2018). Metodología de la investigación. Ciudad de México, México: Mcgraw-Hill Interamericana.

Manzano-Arrondo, V., \& Torrego, L. (2009). Tres modelos para la Universidad. Revista de Educación, (350), 477-489. Recuperado de https://n9.cl/ygf28

Manzano-Arrondo, V. (2011). La universidad comprometida. Barcelona, España: Hegoa.

Manzano-Arrondo, V., \& Suárez, E. (2015). Unidad de Acción Comprometida: una propuesta de solución ante el problema universitario del servicio a la sociedad. Hábitat y Sociedad, (8), 147-165. https://doi. org/10.12795/HabitatySociedad.2015.i8.07

Mestre, U. (2016). Criterios para la evaluación de impacto académico de programas de maestría en modalidad semipresencial, Didasc@lia: didáctica y educación, 7(5), 85-96. Recuperado de https:// dialnet.unirioja.es/servlet/articulo?codigo $=5911162$

Robin, S. (2016). Herramientas de medición del impacto social. Madrid, España: Universidad Oberta de Catalunya.

Scoppetta, O. (2006). Discusión sobre la evaluación de impacto de programas y proyectos sociales en salud pública. Universitas Psychologica, 5(3), 695-703. Recuperado de https://revistas.javeriana.edu.co/ index.php/revPsycho/article/view/471

Teddlie, C., \& Tashakkori, A. (2009). Handbook of Mixed Methods in social and behavioral research. Recuperado de https://n9.cl/39nf

Universidad de Ibagué. (2015). Informe de Renovación de la Acreditación. Programa de Psicología. Manuscrito inédito. 